\title{
Epistemic Network Analysis for Semi-Structured Interviews and Other Continuous Narratives: Challenges and Insights
}

Szilvia Zörgő \& Gjalt-Jorn Ygram Peters

This document is the full text of the article "Epistemic Network Analysis for Semi-Structured Interviews and Other Continuous Narratives: Challenges and Insights", which has been accepted to the first International Conference on Quantitative Ethnography.

The most current version of this manuscript is hosted at PsyArXiv (see doi:10.31234/osf.io/j6n97) - please check the most recent version of the preprint before citing this article.

The $\mathrm{R}$ package described in this manuscript can be installed in $\mathrm{R}$ using, install.packages('rock'); or remotes::install_gitlab('r-packages/rock'); (to install the latest development version; this requires the remotes package to be installed). There is also a GitLab repository and a pkgdown website.

This preprint is licensed under:

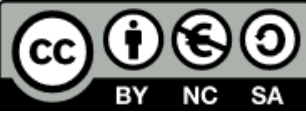




\title{
Epistemic Network Analysis for Semi-Structured Interviews and Other Continuous Narratives: Challenges and Insights
}

\author{
${ }^{凶}$ Zörgő $S^{1}$ and Peters GJY2 \\ ${ }^{1}$ Institute of Behavioural Sciences, Semmelweis University, Budapest, Hungary \\ zorgoszilvialgmail.com \\ ${ }^{2}$ Faculty of Psychology \& Education Science, Open University, Netherlands \\ gjalt-jorn@behaviorchange.eu
}

\begin{abstract}
Applying Quantitative Ethnography (QE) techniques to continuous narratives in an inquiry where manual segmentation with a multitude of codes is preferred poses several challenges. In order to address these issues, we developed the Reproducible Open Coding Kit - convention, open source software, and interface - that eases manual coding, enables researchers to reproduce the coding process, compare results, and collaborate. The ROCK can also be employed to prepare data for Epistemic Network Analysis software. Our paper elaborates the challenges we encountered and the insights we gained while conducting a research project on decision-making regarding therapy choice among patients in Budapest, Hungary. Our aim is to broaden the usage of QE, while facilitating Open Science principles and transparency.
\end{abstract}

Keywords: Epistemic Network Analysis · Semi-structured interviews $\cdot$ Methodology.

\section{$1 \quad$ Introduction}

Epistemic Network Analysis (ENA) has been applied in modelling a wide range of data (1), but continuous narratives, such as semi-structured interviews, are sparse within these worked examples. Continuous narratives are distinguished from discontinuous narratives by the lack of naturally occurring possibilities for segmentation. Due to the fact that ENA is a nascent application, the lack of clear segmentation possibilities needs to be addressed when dealing with continuous narratives in order for ENA to be more broadly utilized. Gaining insight into questions of segmentation is especially important if the project warrants manual segmentation and coding of data.

This paper provides an overview of our project, some of the challenges we encountered and how we addressed these. As a part of weighing options and making decisions regarding 
these challenges, we developed the Reproducible Open Coding Kit (ROCK), which is a protocol and open source software to aid the preparation of data for ENA. ROCK guides the researcher in managing their raw data, performing manual coding and segmentation, as well as aggregating coded data for ENA software.

\section{Research Topic and Relevance}

The manner in which individuals encounter, filter, and process information is vital in decision-making processes; an exemplary domain of said processes is how patients choose a therapy to treat their illness. Decisions regarding healthcare are multifactorial and occur throughout the patient journey.

In Western pluralistic healthcare systems, a frequent distinction is made between conventional and non-conventional medicine, the latter comprised of various modalities referred to as complementary and alternative medicine (CAM). Complementary signifies treatments used in tandem with, alternative connotes treatments employed instead of biomedicine. Most studies agree that CAM use is increasing throughout the Western world (2-4), and depending on the scrutinized illnesses and modalities, ranges between 40-83\% in the United States (5) and reaches 86\% in Europe (6).

Characterizing CAM users poses a challenge. Many authors resort to categorization as a descriptive tool, and "the average CAM user" has been described as a middle-aged, wealthy, well-educated, Caucasian female most likely suffering from cancer $(7,8)$. In addition to problems stemming from introducing such artificial discontinuities (9), there may be a variety of sampling biases (10) and undervalued aspects involved. Clinical factors, such as the type and prognosis of illness, greatly influence choice of therapy $(11,12)$. Furthermore, CAM is often only employed for managing the side-effects of biomedical treatment (13), thus directing attention to the possible differences between motivations in complementary and alternative usage.

The scrutiny of CAM use is clinically significant, as, from a biomedical point of view, it often indicates patient non-compliance and non-adherence. Discontinuing the recommended biomedical treatment may occur in an a priori (refusal to undergo treatment) or a posteriori (discontinuing the treatment) manner (14). Refusal to undergo biomedical treatment or its discontinuation concerning life-threatening illness is often difficult to understand from a physician's perspective. The above phenomena may pose a threat to patient safety and denote tensions in doctor-patient communication, thus warranting thorough analysis.

CAM users are frequently conceptualized as a static, homogeneous group, yet decisions regarding choice of therapy take place throughout the patient journey and are influenced by e.g. novel symptoms, changes in the patient's overall condition, information from the social network of the patient, and the patient's interpretation of these. Because we wanted to explore factors influencing decision-making in a context where little is known of relevant 
cognitive processes and behavior, we chose qualitative methods. Yet we wanted an analytical system that enables us to handle large amounts of data and capture the systemic nature of many variables. A method suited for this is ENA, which allows for the visualization of a large number of variables in a single system. In order to apply ENA, we borrowed terms and concepts from Quantitative Ethnography (QE) that provide for segmentation and data preparation. We also created novel additions to this framework to suit the treatment of our data and continuous narratives in general.

\section{Data Collection}

Our inquiry pre-supposes that patient cognitive and behavioral patterns can be accessed via semi-structured interviews and their scrutiny. The study takes place in Budapest, Hungary, as a continuation of a previous exploratory research project dealing with the same general topic (15). Data collection began in February 2019 and is on-going; at the time of submission, 30 interviews have been conducted with patients, at least another 18 are planned.

Subjects were recruited via convenience sampling, but adhering to a pre-determined quota (see point 4: "Sampling"). Interviews were conducted by four researchers: the principle researcher and three assistants. Assistants were trained in qualitative methods (especially interview techniques), the employed interview structure and code system. Interviews lasted 60 minutes on average, ranging from 40 to 120 minutes; they were soundrecorded and transcribed verbatim. Table 1 shows the main topics of the semi-structured interview, its subtopics, and the number of codes pertaining to these.

Table 1. Areas of the semi-structured interview and related codes.

\begin{tabular}{|l|l|l|l|l|}
\hline $\begin{array}{c}\text { Realm of } \\
\text { inquiry }\end{array}$ & $\begin{array}{c}\text { Topic of } \\
\text { inquiry }\end{array}$ & \multicolumn{1}{|c|}{ Subtopics } & $\begin{array}{c}\text { Question } \\
\text { load }\end{array}$ & \multicolumn{1}{c|}{ Code load } \\
\hline Epistemology & Information & $\begin{array}{l}\text { sources of health- } \\
\text { related information, } \\
\text { appraisal of } \\
\text { information }\end{array}$ & $\begin{array}{l}\text { 6 questions } \\
+ \text { probes }\end{array}$ & $\begin{array}{l}\text { Parent codes }(\mathrm{N}=1) \\
\text { Child codes }(\mathrm{N}=2) \\
\text { Grandchild codes }(\mathrm{N}=16)\end{array}$ \\
\hline Ontology & $\begin{array}{l}\text { Explanatory } \\
\text { Model }\end{array}$ & $\begin{array}{l}\text { concepts of illness } \\
\text { and health; } \\
\text { metaphors of illness } \\
\text { and health }\end{array}$ & $\begin{array}{l}\text { 4 questions } \\
+ \text { probes }\end{array}$ & $\begin{array}{l}\text { Parent codes }(\mathrm{N}=1) \\
\text { Child codes }(\mathrm{N}=2) \\
\text { Grandchild codes }(\mathrm{N}=23)\end{array}$ \\
\hline Behavior & $\begin{array}{l}\text { Patient } \\
\text { journey }\end{array}$ & $\begin{array}{l}\text { choices of therapy, } \\
\text { evaluation of } \\
\text { therapeutic efficacy, } \\
\text { dispositions }\end{array}$ & $\begin{array}{l}5 \text { questions } \\
+ \text { probes }\end{array}$ & $\begin{array}{l}\text { Parent codes }(\mathrm{N}=1) \\
\text { Child codes }(\mathrm{N}=3) \\
\text { Grandchild codes }(\mathrm{N}=13)\end{array}$ \\
\hline
\end{tabular}


For each interview we registered the following: interview date, interviewer ID, interviewee ID, interviewee sex, age, and level of education, diagnosis type (D1-4), specific illness, comorbidities, illness onset, time of diagnosis, and therapy choice (treatment type concerning primary diagnosis: biomedicine only, complementary use of non-conventional medicine, alternative use of non-conventional medicine). For CAM users we also registered type of CAM use (product and/or practitioner, for details see "Sampling" below), attendance in CAM-related courses, disclosure of CAM use to conventional physician and the employed CAM modalities (inductively coded). For users of solely biomedicine, reason for rejecting CAM was deductively coded.

In addition, at the end of each interview, a brief survey containing six questions was administered. Four of these consisted of 3-point Likert scales and inquired about how important "natural" and "holistic" therapies were for the patient, how important it was for their chosen therapy to be "evidenced-based", and whether they make a conscious effort to avoid all pharmaceuticals. Two were open-ended questions and asked participants to define what "natural" and "holistic" meant to them, as these expressions may have vastly different meanings for individuals.

\section{$4 \quad$ Sampling}

Our sampling was based on the primary mode of investigation found within the relevant literature, that is, distinguishing between groups of biomedicine versus CAM users. We utilized non-proportional quota sampling to maximize sample heterogeneity, stratifying on therapy choice (Biomedical and CAM), primary diagnosis (D1, D2, D3, D4), and sex (males and females). Inclusion criteria were the following: 18 years of age or above; having received a diagnosis of D1-D4; and resident of Budapest, Hungary. Our nosological groups were: D1 - Diabetes (I, II, pre-diabetes), D2 - Musculoskeletal diseases, D3 - Digestive illnesses (excluding "sensitivities" and "intolerance"), D4 - Nervous system diseases. Inclusion based on primary diagnosis was defined by the patient retaining a diagnosis of a specific illness (within the given nosology of D1-4) made by a conventional doctor based on biomedical test results.

\subsection{Stratum: Therapy Choice}

\section{Challenges}

Although CAM research scrutinizes biomedicine use vis-à-vis non-conventional medicine use, this distinction implies a false dichotomy. This is sometimes acknowledged by authors who distinguish between three subgroups (biomedicine, complementary, alternative), arriving at the conclusion that these are based on varying patient motivations (16). Yet such research projects are scarce and provide little theoretical guidance for selecting stratification 
criteria. Thus, finding a way to create sampling guidelines and code therapy choice proved to be an important factor in our research design.

\section{Insights}

Aside from our sampling strata, we wanted to take advantage of the ability to explore our data by specifying and comparing a variety of group categorizations post-hoc based on participants' characteristics (i.e. conditional exchangeability). In order to achieve this, we coded patients for sampling groups, but also coded them for other, more detailed choice of therapy: complementary use (CM) or alternative use (AM). Both of these had two subcategories: $\mathrm{CM} / \mathrm{AM}$ through a practitioner or acquisition of products. All of these subgroups may signify distinct cognitive patterns and demarcate different potential subgroups within the sample.

Table 2. Examples for registered participant characteristics enabling conditional exchangeability.

\begin{tabular}{|l|l|l|}
\hline \multicolumn{1}{|c|}{ Category } & \multicolumn{1}{c|}{ Label } & \multicolumn{1}{c|}{ Possible values } \\
\hline Sampling group & GroupID & B or CAM \\
\hline Diagnosis & Diag & D1 or D2 or D3 or D4 \\
\hline Specific illness & Illness & Inductive \\
\hline Treatment type & TreatType & $\begin{array}{l}\text { Biomedicine only (B) / Complementary (CM) / Alternative } \\
\text { (AM) }\end{array}$ \\
\hline CAM use & CAMuse & $\begin{array}{l}\text { Complementary product (CMprod); Complementary } \\
\text { practitioner (CMprac); Alternative product (AMprod); } \\
\text { Alternative practitioner (AMprac) }\end{array}$ \\
\hline
\end{tabular}

\subsection{Stratum: Diagnosis}

\section{Challenges}

Type of illness connotes an important stratum in our sampling because the illness experience, lay theories of illness causation, and the available biomedical cures all interact with it closely and in turn, affect therapy choice. Patients were included based on a primary diagnosis belonging to one of our nosological groups (D1-4). However, patients rarely suffer only from their primary diagnosis, and as such, often discuss or refer to comorbidities as well. Since cognitive and behavioral patterns are likely to vary for different afflictions, it is important to code this information. 


\section{Insights}

We decided that diagnoses needed to constitute a fundamental part of coding so that in later phases of the project, with enough data, we can create distinctive networks based on illnesses and see what kinds of cognitive and behavioral patterns arise. An exhaustive nosological coding tree is unwieldy. Thus, for each participant, we recorded a primary diagnosis (D1-4) and coded the specific illness and comorbidities inductively, classifying them only afterwards. Therefore, a participant received a primary diagnosis code (e.g. D4) and a specific illness (e.g. epilepsy) that was registered at the time of the interview and also coded in the interview transcript. Comorbidities were coded in the transcript (e.g. myoma, migraine) as well, and additionally, symptoms not referring to a specific illness were also coded inductively, as they may be significant in understanding the securitized cognitive processes.

Albeit illnesses signify the only inductive coding in our study, they follow the general structure of all other codes (discussed below). A separate code was designated for narrative segments referring to illness in general.

\section{Operationalization}

\section{$5.1 \quad$ Coding}

Our deductive coding system is based on a qualitative project conducted between Jan. 2015 and June 2017; participant observation was carried out at four sites of Traditional Chinese Medicine (TCM) and 105 patients were involved. Furthermore, semi-structured interviews were conducted with patients and practitioners of TCM $(\mathrm{N}=20)$. The collected data was analyzed with Interpretative Phenomenological Analysis with the aid of Atlas.ti 6.0, and a code system was developed inductively. Our present code system is founded on this previous one; it is comprised of three levels of abstraction, containing 52 low-level codes in total.

\section{Challenges}

The software used for Epistemic Network Analysis requires data in a spreadsheet-like format. However, manually coding transcripts using spreadsheets is not feasible, let alone with such a large number of codes. Completing the binary coding for each utterance would have meant constant sideways scrolling and an inability to view the entire code set; this endeavor would have led to unreliable coding and results. Furthermore, we wanted to code the narratives manually via hermeneutic analysis, which necessitates a full view of each utterance, their proximal context and all applied and applicable codes. 


\section{Insights}

We decided to involve four researchers in the coding process. Three raters were checked for inter-rater reliability vis-à-vis the principal investigator. The coding tasks were divided up amongst the four raters based on the parent codes plus the inductive coding for illnesses. The three research assistants were trained in coding and specialized in detecting their designated parent code and subcodes.

\subsection{Segmentation}

Sentences are the smallest unit of segmentation in our project, they constitute the QE term "utterances". The verbatim transcription of spoken speech comes with inherent subjectivities; as a sentence in speech may persist across vast reaches, the transcriber makes many judgement calls in punctuation. Albeit coding occurs on the level of utterances, cooccurrences are computed based on a higher level of segmentation, the "stanza". Continuous narratives introduce the challenge of defining stanzas as an analytical unit. Stanza size crucially determines analysis results, thus addressing this issue is vital.

\section{Challenges}

Segmentation based on question-response was not an option, as related utterances may persist over several "turns of talk" between interviewer and interviewee. Furthermore, narratives may abruptly digress or terminate. We briefly considered numbering stanzas so that they are linked to each other topic-wise, i.e. interviewees frequently begin a topic, digress, and then return to it again (uptake on a large scale). If so, would stanzas need to receive the same identifier then? We came to the conclusion that determining this would lead to very subjective choices and that we should allow the coding to make this judgement; if stanzas should be linked, they will be linked through code co-occurrence.

\section{Insights}

ENA networks are based on adjacency matrices, where co-occurrence is literal cooccurrence of codes within the same analytical unit (i.e. stanza). Stanza size reflects how much content the researchers consider indicative of psychological proximity. Researchers who are only interested in tightly connected concepts may prefer shorter stanzas, e.g. stanzas may overlap completely with utterances. However, if the research topic concerns broader, more complex issues, researchers may want to define larger stanza sizes to study constructs with higher cognitive distance. Our working definition of a stanza is: a set of one or more utterances that occur in close proximity and discuss the same topic (i.e. recent temporal context). 
Due to the fact that stanzas are so pivotal to the overall network, but our working definition of them is subjective, we decided that discourse segmentation should be performed by three autonomous raters: the principal investigator, a research assistant, and someone not involved in the research project. The latter's unfamiliarity with the questions under scrutiny and employed codes lends a different treatment of the narratives themselves. Albeit coding necessitates specialized knowledge on the part of the rater, the key to optimal discourse segmentation in continuous narratives may be a "naïveté" regarding the subject. Because we want to test this hypothesis, we will retain segmentation from the three raters and run analyses for all to see which set provides the more accurate model; in later phases we might only use one of them or collapse them.

\section{The Reproducible Open Coding Kit (ROCK)}

To summarize our circumstances: we wanted to be able to code and segment our data manually with a multitude of codes and three versions of segmentation. We wanted to employ both deductive and inductive coding in the hermeneutic analysis of continuous narratives. We did not want to work with spreadsheets because accomplishing this would have been problematic. Finally, the large number of participant characteristics (to be used for subgroup exchangeability) required a simpler way of adding this information, sidestepping binary coding. For these reasons, we developed the Reproducible Open Coding Kit (ROCK).

The ROCK is simultaneously a convention for specifying sources of qualitative data and the coding of those sources; it is a Free/Libre Open Source Software R package (17). As such, the ROCK facilitates sharing qualitative coded data, enabling other researchers to reproduce the coding process, compare results, and collaborate by expanding the coding system. The ROCK facilitates adhering to Open Science principles in research, as well as enables transparency and minimizes research waste.

In ROCK terminology, a source is a document that is coded. All sources are stored as plain-text files and consist of one or more utterances, which receive unique utterance identifiers (UIDs) that enable merging or comparing coding by different raters. Utterances are separated by line breaks, and to code an utterance, the relevant code can be appended to the utterance's line in the source file, where each code is delimited by two square opening brackets and two square closing brackets. 
Table 3: Basic ROCK terminology and related functions

\begin{tabular}{|c|c|c|}
\hline Term & Examples and specifics & Function \\
\hline Source & document that is coded (plain-text file) & $\begin{array}{l}\text { delimiting sources of information } \\
\text { (e.g. interviews) and labeling them }\end{array}$ \\
\hline CID & $\begin{array}{l}{[[\text { cid=alice }]]} \\
\text { case identifier used to specify the data } \\
\text { provider (e.g. interviewee) }\end{array}$ & $\begin{array}{l}\text { identifying each participant } \\
\text { separately }\end{array}$ \\
\hline UID & $\begin{array}{l}{[[\text { uid }=73 n t n x 8 n]]} \\
\text { unique identifier for each utterance }\end{array}$ & $\begin{array}{l}\text { Identifying each utterance } \\
\text { separately (which allows, for } \\
\text { example, aggregating information } \\
\text { from various raters for each } \\
\text { utterance) }\end{array}$ \\
\hline $\begin{array}{l}\text { Deductive } \\
\text { code format }\end{array}$ & [[example_code1]] & $\begin{array}{l}\text { providing a universal way of coding } \\
\text { utterances deductively }\end{array}$ \\
\hline $\begin{array}{l}\text { Inductive } \\
\text { code format }\end{array}$ & $\begin{array}{l}{[[\text { examples }>\text { chil } l \mathrm{~d} 1>\operatorname{code} 1]]} \\
\text { hierarchical marker included in code } \\
\text { label }\end{array}$ & $\begin{array}{l}\text { providing a universal way of } \\
\text { specifying hierarchical relationships } \\
\text { between codes while coding } \\
\text { utterances inductively }\end{array}$ \\
\hline $\begin{array}{l}\text { Segmentation } \\
\text { (section } \\
\text { breaks) }\end{array}$ & $\begin{array}{l}\text { <<stanza-delimiter >> } \\
\text { several types of section breaks can be } \\
\text { employed of which "stanza" is one }\end{array}$ & $\begin{array}{l}\text { Parsing the narrative into groups of } \\
\text { utterances }\end{array}$ \\
\hline Attributes & $\begin{array}{l}\text { rock_attributes: } \\
\quad- \\
\text { caseId: } 1 \\
\quad \text { gender: female } \\
\text { age: } 50 \mathrm{~s} \\
\text {--- }\end{array}$ & $\begin{array}{l}\text { Providing additional information } \\
\text { about cases, such as metadata about } \\
\text { data collection or characteristics of } \\
\text { participants. }\end{array}$ \\
\hline
\end{tabular}

The ROCK supports both deductive and inductive coding. Inductive codes use the "greaterthan" sign $(>)$ as hierarchical markers to signify parent-child relationships, e.g.: [[examples $>$ code1]]. The rock $\mathrm{R}$ package can read all codes and reconstruct the inductive code tree.

In addition to coding functionality, the ROCK allows specification of dynamic sets of identifiers, e.g.: a case identifier (CID). CIDs can be used to specify the data provider of sets of utterances, for example by specifying which individual said what. The utterances identifier by a given case identifier can then easily be supplemented with the attributes specified for that case (e.g. metadata or demographic variables). Finally, the ROCK allows specifying sections in each source. Sections can be automatically counted and numbered, 
providing easy means to segment sources (e.g.: into stanzas) using a variety of segmentations in tandem.

To perform coding and segmentation, we developed iROCK, a simple online interface consisting of a file that combines HTML, CSS, and javascript to provide a rudimentary graphical user interface. Because iROCK is a standalone file, it does not need to be hosted on a server, which means that no data processing agreements are required (as per the GDPR). The iROCK interface allows raters to upload a source, a list of codes, and segmentation identifiers; coding consists of dragging and dropping codes upon utterances at the end of their line (see Figure 1 for illustration). Once coding is finished, the coded sources can be saved. The rock $\mathrm{R}$ package then reads these and constructs a dataframe in $\mathrm{R}$, which is then ready for further processing by the rENA R package or for export to a comma separated values file that the ENA web application can use.

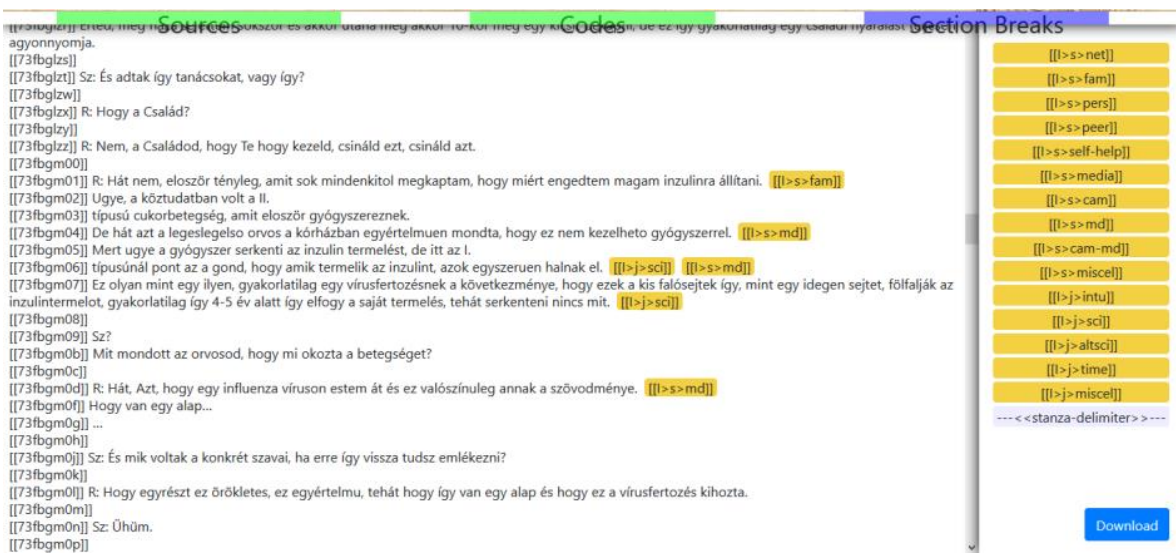

Figure 1: The iROCK interface. The top ribbon displays controls for uploading sources, codes, and section breaks. The panel on the right contains the uploaded codes that are appended to the chosen utterance on the left. The narrative is parsed according to utterances, each receives a unique identifier. (The coded fragment is merely illustration.)

\section{Our application of ROCK}

First, all interview transcripts were stored as plain-text files with the rock extension. Because all interviews were individual interviews, the first line of every source was labelled with a CID that linked that source's utterances to that case. Second, the sources were "cleaned" by the rock R package (e.g. indicating utterances by inserting newline characters at appropriate places), and the cleaned versions were stored in a different directory. Third, every utterance received a unique UID, and the resulting versions were stored in yet another directory. This process documents each step, enabling inspection of intermediate steps if required at any point. Codes (3 lists of subcodes based on their parent) 
and 3 ways of denoting segmentation (professional, middle, and "naïve") were created and placed into separate plain-text files, with the attributes for each source listed in a separate plain-text file. Raters import sources, codes, segmentation identifiers into iROCK, perform coding, then download their document into a designated folder. The rock $\mathrm{R}$ package collapses all information within the folders based on UID and can then construct $\mathrm{R}$ dataframes.

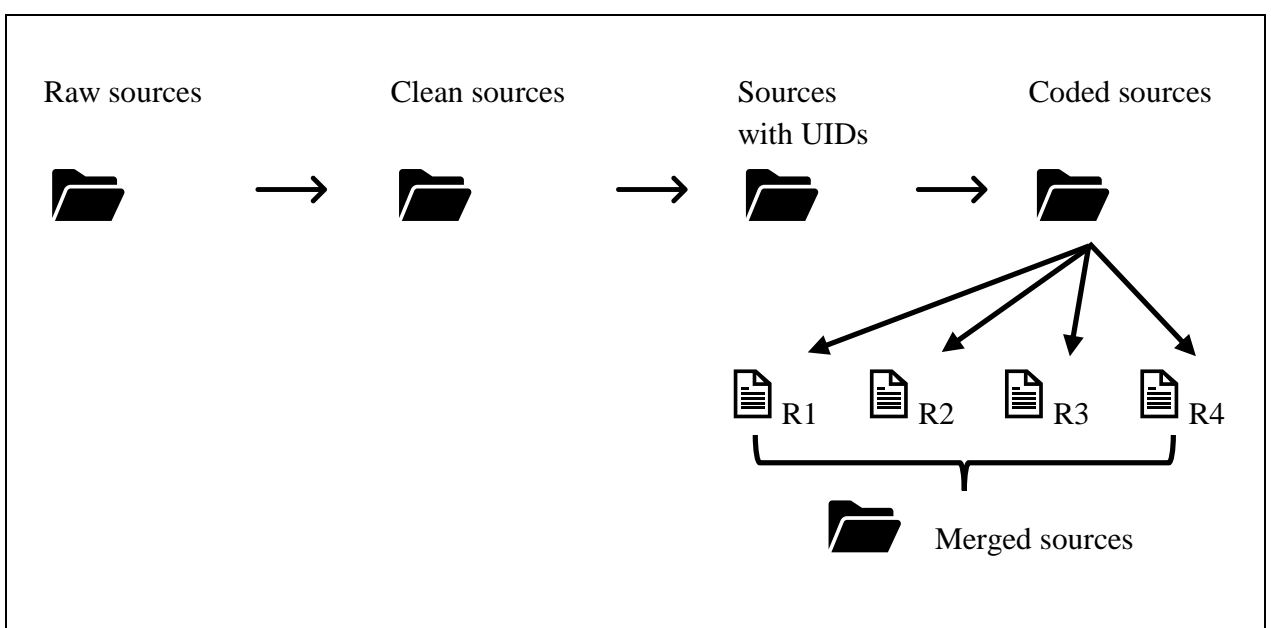

Figure 2: This flowchart shows the process of cleaning and merging sources. The first folder (Raw sources) contains the transcripts in plain text documents. The second folder (Clean sources) contains the narratives parsed according to one utterance per line. The third folder (Sources with UIDs) contains the parsed narratives where each line receives a unique identifier. These sources are uploaded to iROCK where coding and segmentation are performed. The last folder (Coded sources) contains the parsed, coded narratives downloaded from iROCK. The coded sources from our four researchers (R1-4) are then merged based on UIDs, thus creating master documents with sources containing all codes for each utterance. Merged sources can then be converted by the ROCK to qualitative data tables and uploaded to the ENA interface.

\section{$7 \quad$ Closing Remarks}

We hope the disclosure of our challenges and means of addressing them offers insight into QE techniques applied to continuous narratives. Furthermore, the aim of this pilot study is to operationalize the ROCK and make it available for use by other researchers as well. 


\section{Acknowledgements}

The authors would like to acknowledge the support of ÚNKP-18-3-III New National Excellence Program of the Ministry of Human Capacities, Hungary. We would also like to thank Brendan Eagan for his valuable insights throughout the planning and implementation phases of this research. Lastly, we are grateful to research assistants Anna Geröly, Anna Jeney, and Krisztina Veres for their rigorous work.

\section{References}

1. Shaffer D (2018) Epistemic network analysis: Understanding learning by using big data for thick description. In: International Handbook of the Learning Sciences., Fischer, F., Hmelo-Silver, C. E., Goldman, S. R., Reimann, P. (Eds.). Routledge, New York, pp 520-531

2. Frass M, Strassl R, Friehs H, Müllner M, Kundi M, Kaye A (2012) Use and acceptance of complementary and alternative medicine among the general population and medical personnel: a systematic review. Ochsner J 12:45-56

3. Thomas K, Nicholl J, Coleman P (2001) Use and expenditure on complementary medicine in England: a population based survey. Complement Ther Med 9:2-11

4. Tindle H, Davis R, Phillips R, Eisenberg D (2005) Trends in use of complementary and alternative medicine by US adults: 1997-2002. Altern Ther Health Med 11:429

5. Arthur K, Belliard J, Hardin S, Knecht K, Chen C, Montgomery S (2012) Practices, Attitudes, and Beliefs associated with Complementary and Alternative Medicine (CAM) use among Cancer Patients. Integr Cancer Ther 11:232-42

6. Eardley S, Bishop F, Prescott P, Cardini F, Brinkhaus B, Santos-Rey K, Vas J, von Ammon K, Hegyi G, Dragan S, Uehleke B, Fønnebø V, Lewith G (2012) A systematic literature review of complementary and alternative medicine prevalence in EU. Forsch Komplementmed 19(Suppl 2):18-28

7. Harris P (2012) Prevalence of complementary and alternative medicine (CAM) use by the general population: a systematic review and update. Int J Clin Pract 66:92439 
8. Ernst E (2000) Prevalence of use of complementary/alternative medicine: a systematic review. Bulletin of the World Health Organization 78:

9. Altman D (1994) Problems in dichotomizing continuous variables. Am J Epidemiol $15: 442-5$

10. Henrich J, Heine S, Norenzayan A (2010) The weirdest people in the world? Behav Brain Sci 33:61-83

11. Faith J, Thorburn S, Tippens K (2013) Examining CAM use disclosure using the behavioral model of health services use. Complement Ther Med 21:501-8

12. Thomson P, Jones J, Browne M, Leslie S (2014) Psychosocial factors that predict why people use complementary and alternative medicine and continue with its use: A population based study. Complementary Therapies in Clinical Practice 20:302310

13. Stratton T, McGivern-Snofsky J (2008) Toward a sociological understanding of complementary and alternative medicine use. J Altern Complement Med 14:777-83

14. Zörgő S, Olivas Hernández O (2018) Patient Journeys of Nonintegration in Hungary: A Qualitative Study of Possible Reasons for Considering Medical Modalities as Mutually Exclusive. Integrative Cancer Therapies 17:1270-1284

15. Zörgő S, Purebl G, Zana Á (2018) A Qualitative Study of Culturally Embedded Factors in Complementary and Alternative Medicine Use. BMC Complementary and Alternative Medicine

16. Astin J (1998) Why patients use alternative medicine: results of a national study. JAMA 279:1548-53

17. Peters G, Zörgő S (2019) Introduction to the Reproducible Open Coding Kit (ROCK). Psyarxiv. doi: https://doi.org/10.31234/osf.io/stcx9 